\title{
MPTP Neurotoxicity and Testosterone Induce Dendritic Remodeling of Striatal Medium Spiny Neurons in the C57B1/6 Mouse
}

\author{
Eleni Antzoulatos, ${ }^{1,2}$ Michael W. Jakowec, ${ }^{3}$ Giselle M. Petzinger, ${ }^{3}$ and Ruth I. Wood ${ }^{2}$ \\ ${ }^{1}$ Neuroscience Graduate Program, University of Southern California, Los Angeles, CA 90033, USA \\ ${ }^{2}$ Department of Cell and Neurobiology, Keck School of Medicine, University of Southern California, Los Angeles, \\ CA 90033, USA \\ ${ }^{3}$ Department of Neurology, Keck School of Medicine, Department of Biokinesiology, University of Southern California, Los Angeles, \\ CA 90033, USA
}

Correspondence should be addressed to Ruth I. Wood, riw@usc.edu

Received 11 September 2010; Accepted 27 March 2011

Academic Editor: Katerina Venderova

Copyright () 2011 Eleni Antzoulatos et al. This is an open access article distributed under the Creative Commons Attribution License, which permits unrestricted use, distribution, and reproduction in any medium, provided the original work is properly cited.

\begin{abstract}
Nigrostriatal damage is increased in males relative to females. While estrogen is neuroprotective in females, less is known about potential protective effects of testosterone in males. We determined if castration enhances neuronal injury to 1-methyl-4phenyl-1,2,3,6-tetrahydropyridine (MPTP). Castrates or sham-castrated mice were sacrificed 1 week following injection of MPTP $(4 \times 20 \mathrm{mg} / \mathrm{kg})$ or saline $(n=11-12 /$ group$)$. The right striatum was immunostained for tyrosine hydroxylase $(\mathrm{TH})$. The left hemisphere was stained by Golgi Cox to quantify neuronal morphology in medium spiny neurons (MSNs) of the dorsolateral striatum. MPTP reduced TH, but there was no effect of castration and no interaction. For MSN dendritic morphology, MPTP decreased the highest branch order and increased spine density on 2nd-order dendrites. Castrated males had shorter 5th-order dendrites. However, there was no interaction between gonadal status and MPTP. Thus, castration and MPTP exert nonoverlapping effects on MSN morphology with castration acting on distal dendrites and MPTP acting proximally.
\end{abstract}

\section{Introduction}

Gonadal steroid hormones are potent modulators of neuronal survival and neuronal morphology [1]. In the adult, steroid hormones exert activational effects in steroid-responsive brain regions which include protective effects against neurodegeneration $[2,3]$. However, sex differences exist in many neurodegenerative disorders, suggesting that the male and female brains are not equally responsive to gonadal steroids.

In this regard, Parkinson's disease (PD) is a common neurological disorder that demonstrates a substantial sex difference, with a one- to twofold higher incidence in men [4]. PD results from the progressive loss of dopaminergic neurons in the substantia nigra pars compacta (SNC; [5]). Dopaminergic efferents from SNC project rostrally as part of the nigrostriatal pathway to the dorsolateral striatum, where they synapse onto medium spiny neurons (MSNs). These efferents are reduced in PD, leading to a depletion of striatal dopamine. Gonadal steroids modulate the function of the nigrostriatal system and are thought to contribute, in part, to this sex difference.

In females, estrogen promotes the function of the nigrostriatal system by enhancing striatal dopamine release, increasing dopamine metabolism and altering both dopamine receptors and uptake sites $[6,7]$. In response to neurotoxic insult, estrogen is also neuroprotective in the nigrostriatal system [7]. This has been well demonstrated using 1-methyl-4-phenyl-1,2,3,6-tetrahydropyridine (MPTP) and 6-hydroxydopamine (6-OHDA), neurotoxins which selectively deplete dopaminergic SNC neurons [8]. In both models, estrogen attenuates the loss of striatal dopamine and reduces the loss of SNC dopaminergic neurons [9-15]. 
In parallel to its effects in the female, estrogen also modulates nigrostriatal function in males [16, 17]. Estrogen in males is derived by the local aromatization of testosterone, although testosterone can also act as an androgen in the male brain. Whether testosterone has neuroprotective effects in the male nigrostriatal system, similar to the effects of estrogen in the female, is not well-established. After MPTP in castrated mice, striatal dopamine loss is attenuated by estrogen but not by testosterone $[18,19]$.

Previous studies investigating the effects of testosterone on nigrostriatal function in male rats and mice after 6OHDA or MPTP have measured dopamine content and release [18-21], dopaminergic striatal input [21], and dopamine transporter binding $[19,21]$. It is unknown whether testosterone reduces the loss of dopaminergic innervation to the striatum after MPTP in mice. Ultimately, the effects of MPTP lesion may also extend beyond the SNC dopamine neurons themselves. Loss of dopaminergic input may remodel MSN morphology, and testosterone has potential to attenuate this MPTP-induced deafferentation. The current study used castrated and gonad-intact adult male mice to determine whether testosterone reduces MPTPinduced deficits in striatal dopamine neurochemistry and MSN neuronal architecture.

\section{Materials and Methods}

2.1. Animals. Forty-five C57BL/6 adult male mice (8-10 weeks old) were obtained from Charles River Laboratories (Wilmington, MA). Mice were group-housed on a 12:12 LD photoperiod with access to food and water ad libitum. Experimental procedures were approved by USC's Institutional Animal Care and Use Committee and conducted in accordance with the NIH Guide for the Care and Use of Laboratory Animals (DHEW Publication 80-23, revised 1985, Office of Science and Health reports, DRR/NIH, Bethesda, MD).

Initially, half of the mice $(n=23)$ were castrated (OrchX) via a midline scrotal incision. The other half $(n=$ 22) received sham castrations. As measured by androgensensitive seminal vesicle weight, castration was effective $(187.5 \pm 14.2 \mathrm{mg}$ in sham males versus $5.6 \pm 1.4 \mathrm{mg}$ in castrated males, $P<.05)$. Two weeks later, half of the mice in each group received MPTP and half were given saline. MPTP (Sigma, St. Louis, MO) was dissolved in $0.9 \%$ saline and was administered in 4 injections of $20 \mathrm{mg} / \mathrm{kg}$ (free-base).ip. with an interinjection interval of 2 hours. Control mice received equivalent injections of $0.1 \mathrm{~mL}$ saline. This lesioning paradigm is a well-established method that leads to ca. $67 \%$ loss of nigrostriatal neurons and $90-95 \%$ depletion of striatal $\mathrm{DA}$, as reported in previous studies from our laboratories $[22,23]$.

One week following MPTP, animals were sacrificed via intracardiac perfusion. This duration is sufficient for MPTP-induced cell death to occur [22]. Mice were deeply anesthetized with sodium pentobarbital $(150 \mathrm{mg} / \mathrm{kg}$ BW $)$ and perfused intracardially with $150 \mathrm{~mL}$ of $0.1 \mathrm{M}$ sodium phosphate buffer $(\mathrm{PB}, \mathrm{pH}=7.4)$ containing $0.9 \% \mathrm{NaCl}$ and
$0.1 \% \mathrm{NaNO}_{3}$. The brains were removed and hemisected. To allow us to obtain TH and neuronal morphologic measures in the same animals, the right hemisphere for each brain was processed for TH immunocytochemistry and the left hemisphere was processed for Golgi-Cox staining. We are unaware of any evidence of laterality in striatal damage after.ip. MPTP injections in the mouse.

\subsection{Tyrosine Hydroxylase Immunocytochemistry. The right} hemispheres from each brain were postfixed in $4 \%$ paraformaldehyde in $\mathrm{PB}$ overnight at $4^{\circ} \mathrm{C}$, then cryoprotected for 5 days at $4^{\circ} \mathrm{C}$ with $20 \%$ sucrose in $\mathrm{PB}$. Hemispheres were rapidly frozen and sectioned coronally at $25 \mu \mathrm{m}$ thickness through the rostrocaudal extent of the striatum. Sections were stored in $\mathrm{PB}$ with $0.01 \%$ sodium azide at $4^{\circ} \mathrm{C}$ until processed for TH immunocytochemistry.

Sections through the striatum at or rostral to the anterior commissure corresponding to Plates 18-28 of Paxinos and Franklin [24] were stained for TH. Tissue from mice in different groups was stained at the same time. Sections were incubated overnight at room temperature (RT) in polyclonal rabbit anti-TH antibody $(1: 5000$; Chemicon, Temecula, CA) with $4 \%$ normal donkey serum and $0.3 \%$ Triton X100 in PB. The following day, sections were incubated in biotinylated donkey antirabbit secondary antibody $(1: 200$; Jackson Immunoresearch, West Grove, PA) and the avidinbiotin-horseradish peroxidase complex (Vector Elite Kit; Vector Laboratories, Burlingame, CA), each for 1 hour at RT with extensive washes in between. TH-labeled cells were visualized using NiCl-enhanced 3',3-diaminobenzidine tetrahydrochloride with $0.25 \%$ hydrogen peroxide. Sections were mounted onto gelatin-coated slides, dehydrated in alcohols, cleared in xylenes, and coverslipped with Permount.

The relative expression of $\mathrm{TH}$ immunoreactivity was measured in dorsolateral striatum on coded slides by an observer blind to the treatment group. To ensure that differences in staining intensity were due to differences in antigen expression, multiple sections from each of the different treatment groups were handled concurrently in identical staining conditions. Control experiments excluding either primary or secondary antibody were also carried out to verify staining specificity. Three striatal sections rostral to the anterior commissure (Bregma 0.25-1.25 in [24]) were sampled per animal ( $n=8-9$ animals/group) using methods previously described by our laboratory $[25,26]$. Briefly, striatal sections were digitally photographed at low magnification. The dorsolateral quadrant of each striatal section was outlined, and TH immunostaining was measured in a $1.6 \mathrm{~mm}^{2}$ circular region of interest at the dorsolateral boundary of this quadrant (Figure 1). In previous studies $[25,26]$, this region shows the largest decrease in $\mathrm{TH}$ immunostaining after MPTP. The relative optical density (expressed as arbitrary units within the linear range of detection) was determined by subtracting the relative optical density of the corpus callosum as background. This measurement reflects both the area and intensity of $\mathrm{TH}$ immunostaining within the striatum. To ensure that the gray values represented an optical density within the nonsaturated 


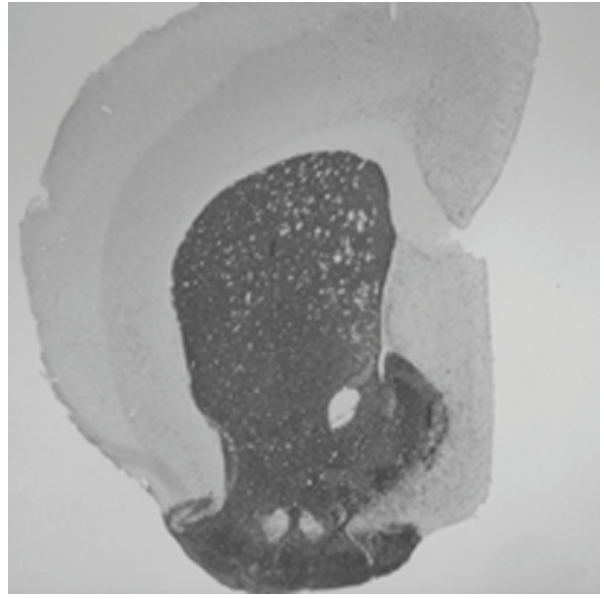

(a)

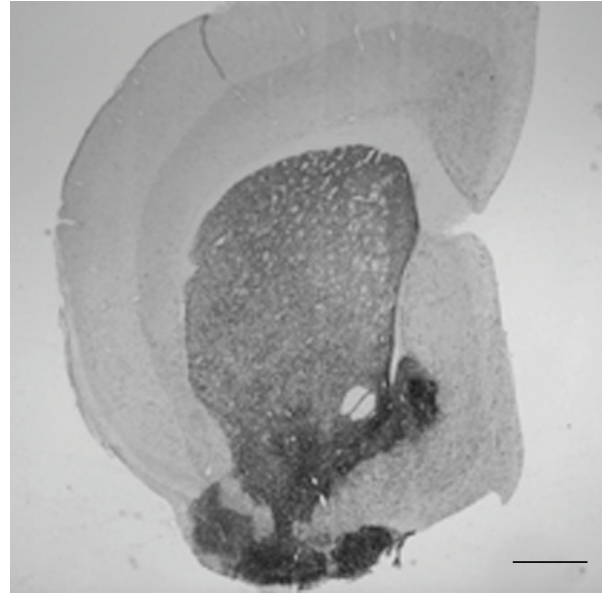

(b)

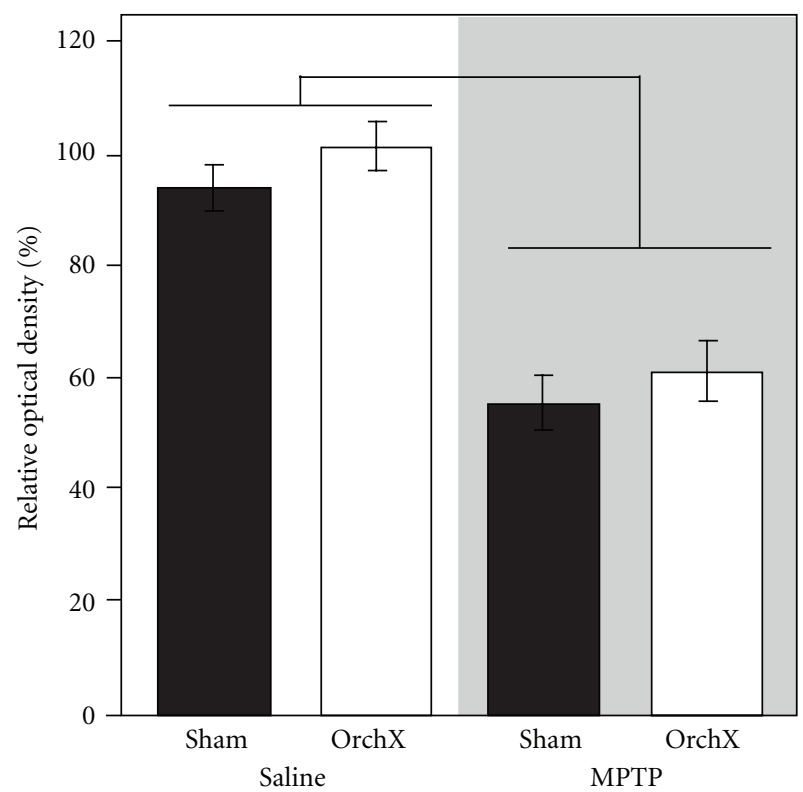

(c)

FIGURE 1: TH staining in the dorsolateral striatum. Photomicrographs of TH staining in gonad-intact saline-injected (a) and MPTP-treated (b) mice. Scale bar $=500 \mu \mathrm{m}$. (c) The density of TH staining in the dorsolateral striatum of saline-injected (clear) and MPTP-treated (shaded) gonad-intact (black bars) and orchidectomized (white bars) male mice ( $n=8-10$ mice/group). The bar represents an effect of MPTP $(P<.05)$. OrchX: orchidectomized; Sham: sham-orchidectomized.

range of the image analysis, a Kodak photographic step tablet (density range to $255 \mathrm{OD}$ units) captured by the CCD camera was used. Maximal tissue immunostaining relative OD units did not exceed the relative OD units of the tablet.

2.3. Golgi-Cox Staining. Golgi-Cox staining was performed on the left hemisphere of each brain using the FD Rapid GolgiStain Kit (FD NeuroTechnologies, Ellicott City, MD). Pilot studies using Golgi staining (according to Gomez and Newman [27]), Golgi-cox staining (according to Gibb and Kolb [28]), and the rapid Golgi kit (FD NeuroTechnologies) were conducted to determine an optimal way to visualize neuronal morphology in our striatal tissue. The rapid Golgi kit provided the most complete staining of medium spiny neurons.

The hemispheres were placed in Golgi-Cox solution containing mercuric chloride, potassium dichromate, and potassium chromate for 2 weeks, and the solution was replaced after the first 24 hours. The brains were moved to a cryoprotection solution (GolgiStain Kit) for 48 hours and then sectioned coronally at $200 \mu \mathrm{m}$ on a vibratome (Vibratome Series 1000). Sections through the rostral-caudal extent of the striatum were mounted on gelatin-coated slides. Slides were stored in a humidity chamber overnight and developed the following day according to the Rapid GolgiStain Kit protocol. Briefly, slides were rinsed in distilled water and placed in a developing solution for 10 minutes. 
Immediately afterwards, the slides were rinsed, dehydrated in alcohols, cleared in xylenes, and coverslipped with Cytoseal60 mounting medium (Richard-Allan Scientific). Slides were stored in the dark at RT until morphological analysis.

MSN morphology was analyzed on coded slides by an observer blinded to the treatment groups using a Nikon Eclipse 80i microscope (Nikon Instruments, Inc., Melville, NY) with motorized stage and MicroFire camera (Olympus America, Inc., Center Valley, PA). To compare dendritic morphology after castration and MPTP lesion, the entire dendritic tree from one primary dendrite was traced under a 100x oil immersion lens using the Neuron Tracing function in NeuroLucida (MicroBrightField,Inc., Williston, VT). Brains with well-differentiated Golgi-Cox labeling from 5 mice in each experimental group were selected for morphologic analysis; 5 neurons from each mouse were analyzed. Morphologic data from the 5 neurons/mouse were averaged to provide a single data point for each animal used in statistical comparison ( $n=5$ /group). MSNs selected for analysis were located in the dorsolateral quadrant of the striatum at or rostral to the level of the anterior commissure (Plates 18-28 of [24]). Selected MSNs were fully impregnated with Golgi stain and had clearly visible spines with minimal or absent obstruction by neighboring Golgi-stained cells or blood vessels. Morphometric analysis was conducted using NeuroExplorer software (MicroBrightField, Inc.). Briefly, each dendritic segment was assigned a branch order with the dendritic segment proximal to the soma identified as the first branch order. Dendritic lengths, number of spines, and spine density were computed for each branch order. All dendrites subject to morphologic analysis had at least 3 branch orders. However, because not all dendrites had 4th- and 5th-order branches, the variability in dendritic length increased at higher branch orders. In addition, total spine density and total dendrite length were calculated for the entire dendritic tree. Due to the relative lack of spines on primary dendrites (typically, 1 or 2 spines/primary dendrite), branch order analysis was not performed on first-order dendrites.

2.4. Statistics. For comparison of both $\mathrm{TH}$ and GolgiCox labeling, morphologic data from each mouse were averaged to provide a single data point used in statistical comparison. Group differences for the 5 animals in each group were analyzed by two-factor (gonadal status and lesion) analysis of variance (ANOVA). Post hoc comparisons using the Fisher's LSD test were conducted when statistically significant differences $(P<.05)$ were found.

\section{Results}

3.1. Striatal TH. Striatal TH immunocytochemistry was used here as a measure of lesion damage in gonad-intact and castrated males. Damage to midbrain DA neurons causes a loss of striatal DA terminals, which produces equivalent changes in striatal levels of presynaptic dopamine transporter and $\mathrm{TH}[23,29,30]$. Similar to previous reports by our lab and others $[25,26,31,32]$, MPTP decreased $\mathrm{TH}$ immunoreactivity in the striatum (Figures 1 (a) and 1(b), $n=$ 8-10/group). Striatal TH was reduced by $40 \%$ after MPTP (mean gray level $59.4 \pm 3.7$ versus $98.5 \pm 3.1$ in unlesioned mice, $F(1,31)=64.883, P<.05$, Figure $1(\mathrm{c}))$. However, there was no effect of castration on $\mathrm{TH}$ immunoreactivity and no interaction between MPTP and castration $(P>.05$, Figure $1(\mathrm{c}))$. This finding parallels previous studies which have found no effect of castration on the number of $\mathrm{TH}$ positive neurons in SNC [33].

\subsection{Medium Spiny Neuron Morphology}

3.2.1. Spine Density. MSNs have elaborate dendritic arbors with a high density of dendritic spines (Figure 2). In saline-treated sham-castrate controls, spines were largely absent from primary dendrites $(1.2 \pm 0.2$ spines $/ 10 \mu \mathrm{m})$, but increased on more distal dendrites (5th-order dendrites $6.8 \pm$ 0.4 spines $/ 10 \mu \mathrm{m})$. The density of dendritic spines in the present study ( $n=5$ /group) is comparable to that reported previously in mice [34-36]. When combining castrated and gonad-intact mice, MPTP produced a modest but significant increase in total spine density (spines/10 $\mu \mathrm{m})$ on MSNs $(6.9 \pm$ 0.1 versus $6.3 \pm 0.1$ in saline-injected castrate and intact mice, $F(1,16)=10.22, P<.05$, Figure $3(\mathrm{a}))$. When analyzed according to branch order, the increase in spine density was restricted to proximal dendrites (Figure 4(a)). Specifically, MPTP-treated mice had a higher spine density on 2nd-order $(5.3 \pm 0.3)$ and 3 rd-order $(7.1 \pm 0.2)$ dendrites, compared with $4.2 \pm 0.2$ and $6.5 \pm 0.2$ in saline-injected mice, respectively $(F(1,16)=9.659$ and 5.700, $P<.05$, Figure 4(a)). However, there was no effect of castration on spine density and no interaction $(P>.05$, Figures $3(\mathrm{a})$ and $4(\mathrm{a}))$.

3.2.2. Branch Order. MPTP significantly decreased the average highest branch order $(3.9 \pm 0.1)$ compared to salineinjected males $(4.3 \pm 0.1, F(1,16)=4.595, P<.05$, Figure 3(b)). As with other measures of overall neuronal morphology, castration was without effect and there was no interaction (Figure 3(b)).

3.2.3. Dendritic Length. There was no effect of MPTP on total dendrite length $(402.0 \pm 17.4 \mu \mathrm{m}$ versus $415.5 \pm 20.2 \mu \mathrm{m}$ in saline-injected mice, $P>.05$, Figure 3(c)). However, in parallel to the increase in spine density, we observed a selective increase in dendritic length after MPTP on 2nd-order dendrites $(80.2 \pm 6.8 \mu \mathrm{m}$ versus $61.6 \pm 4.6 \mu \mathrm{m}$ in saline-injected mice, $F(1,16)=4.796, P<.05$, Figure $4(\mathrm{~b}))$. Castration had no effect on total dendritic length. However, castrated mice had a prominent reduction in dendritic length of distal dendrites. Specifically, 5th-order dendritic length was significantly reduced in castrated mice $(78.9 \pm 14.8 \mu \mathrm{m})$ compared with gonad-intact males $(138.3 \pm$ $13.2 \mu \mathrm{m}, F(1,16)=6.659, P<.05$, Figure $4(\mathrm{~b}))$. There was no interaction between MPTP treatment and gonadectomy (Figures 3(c) and 4(b)). 


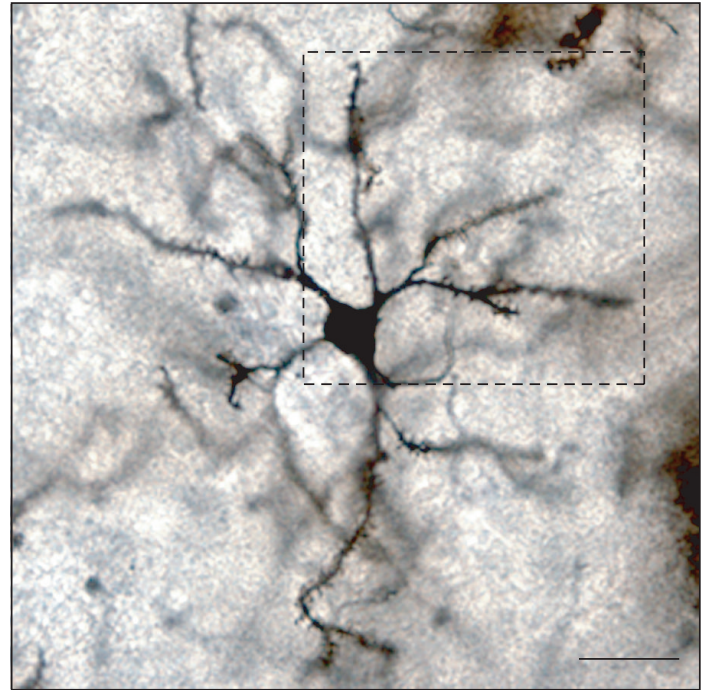

(a)

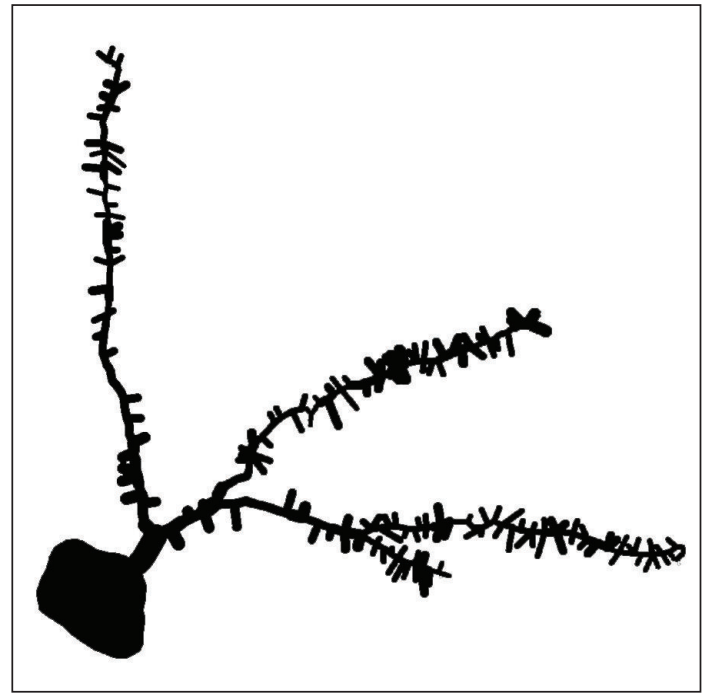

(b)

FIgURE 2: Golgi stained MSNs. Photomicrograph of a representative Golgi-impregnated medium spiny neuron with high-magnification inserts of a primary dendritic branch and fifth-order dendritic branch (a) and corresponding neurolucida tracing (b). Scale bar $=10 \mu \mathrm{m}$.

\section{Discussion}

The current study used the MPTP mouse model of PD to investigate the effects of castration on dopamine-depleting lesions of the nigrostriatal system. MPTP decreased striatal $\mathrm{TH}$ immunoreactivity, reduced the average highest branch order on MSNs, and increased proximal spine density. Separately, castration reduced dendritic length of distal dendrites. We predicted that testosterone would act as a neuroprotectant to attenuate the effects of MPTP and that castrated mice would have increased nigrostriatal damage after MPTP compared with gonad-intact mice. However, there was no interaction between gonadal hormone status and MPTP, suggesting that testosterone does not attenuate the neurotoxic effects of MPTP in the nigrostriatal system of males.

The current study used a well-established lesioning protocol $[22,23]$ that produced a moderate lesion, as measured by TH immunostaining. This is relevant to the study of how gonadal steroid hormones act on the nigrostriatal system because the neuroprotective effects of gonadal hormones are likely to be evident earlier in PD. For example, PD symptom severity is sexually dimorphic in early stages of the disease, with women experiencing less severe motor impairments [4, 37, 38]. This has been attributed, in part, to the neuroprotective effects of estrogen on the nigrostriatal system. In later stages of $\mathrm{PD}$, sex differences are not reported, presumably because severe nigrostriatal degeneration obscures the effects of neuroprotective factors, including estrogen. This is paralleled in animal studies, where moderate lesions do not overwhelm the potential for gonadal hormones to attenuate the nigrostriatal response to MPTP. In 6-OHDA- lesioned rats, Gillies et al. [21] have demonstrated sex differences with small doses of 6-OHDA that disappear with larger doses. Using MPTP, our laboratory has demonstrated sex differences in motor impairments after relatively small lesions [39]. Even so, in the current study, castration did not alter the morphologic response to MPTP. Larger lesions should produce a more dramatic depletion of TH, but seem unlikely to reveal an interaction with castration.

Nonetheless, our results do demonstrate a broad impact of MPTP on basal ganglia circuitry. Although MPTP selectively kills dopamine-producing neurons of SNC, its effects are not limited to dopaminergic neurons themselves. In fact, morphological effects of MPTP were observed on efferent targets in the striatum. Specifically, MPTP increased dendritic spine density on proximal dendrites of striatal MSNs. This result was initially surprising because loss of dopaminergic input to MSNs is expected to reduce spine density, at least as demonstrated in vitro $[34,40]$. Importantly, the increased spine density observed in our study was restricted to proximal dendrites. Proximal MSN dendrites receive synaptic inputs from within the striatum, while distal dendrites receive extrinsic inputs from the cortex and SNC [41]. This suggests that the increase in proximal dendritic spine density after MPTP is driven by intrinsic striatal neurons, rather than dopaminergic neurons of SNC. This may reflect a compensatory reaction to dopamine depletion and demonstrates the dynamics of spine morphology with degree of lesion, model, and time postlesion.

Striatal MSNs receive a variety of afferent inputs which include glutamatergic inputs from cortex as well as dopaminergic projections from SNC. Interestingly, MSN spine density is increased after methamphetamine, which also depletes striatal dopamine [42]. However, the methamphetamineinduced increase in spine density is selective to distal dendrites [43-45], while the effect that we observed occurred proximally. Given the importance of dopaminergic projections to the striatum, the absence of MPTP-induced 


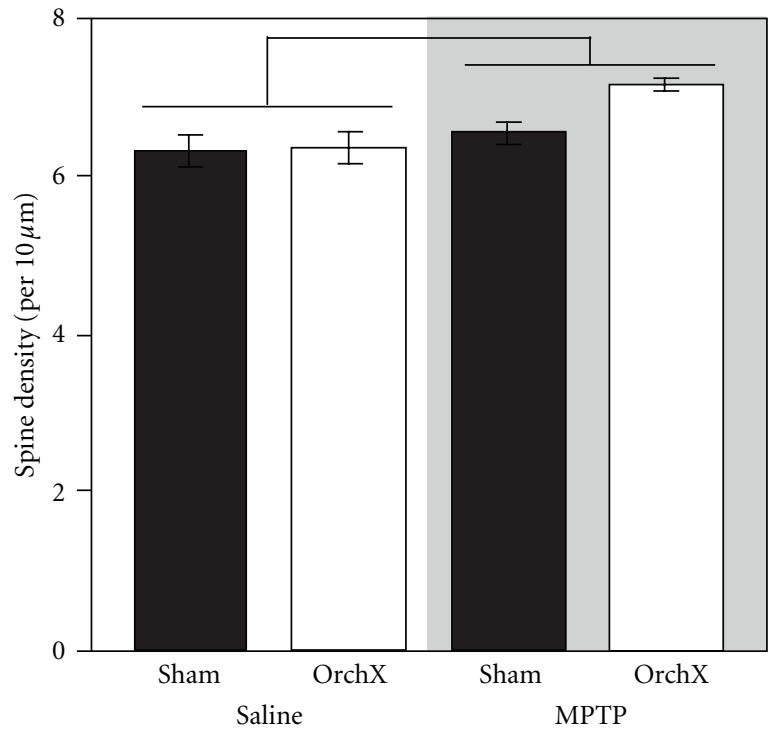

(a)

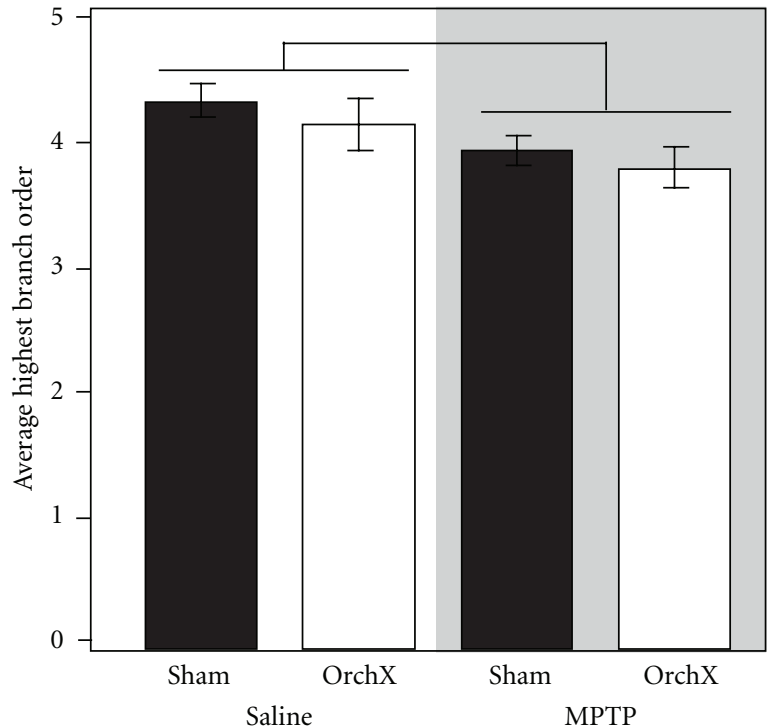

(b)

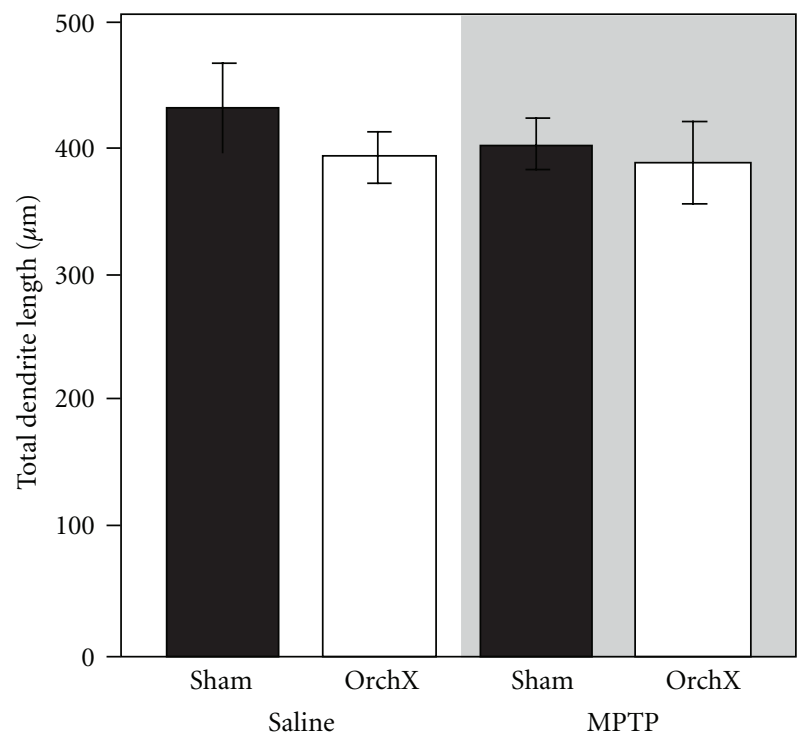

(c)

Figure 3: Neuronal morphology totals. Total spine density (a), average highest branch order (b), and total dendrite length (c) in salineinjected (unshaded panel) and MPTP-treated (shaded panel) gonad-intact (black bars) and orchidectomized (white bars) male mice ( $n=$ 5 mice/group). Bars represent an effect of MPTP $(P<.05)$. OrchX: orchidectomized; Sham: sham-orchidectomized.

structural changes to MSN distal dendritic spines is indeed unexpected.

The absence of MPTP-induced structural changes to distal dendrites may relate to the distribution of striatal MSNs and the heterogeneity of striatal structure. MSNs represent a heterogeneous population comprised of both D1 receptorcontaining neurons of the direct, striatonigral pathway and D2 receptor-containing neurons of the indirect, striatopallidal pathway [41]. The indirect pathway has also been implicated behaviorally, with D2 receptor knockout mice exhibiting PD-like akinesia and bradykinesia [46]. Behavioral deficits in D1 receptor knockout mice are minimal [47] or absent [48]. Recently, Day et al. [34] demonstrated a selective effect of dopamine-depleting lesions on D2 receptor containing MSNs using 6-OHDA. Therefore, it is possible that MPTP-induced spine changes are also confined to the D2 receptor-containing subpopulation of MSNs.

The other key observation from our study was that castrated and gonad-intact males had the same response to MPTP, suggesting that testosterone fails to protect against MPTP-induced neuronal damage. Initially, we postulated that testosterone would be neuroprotective in males, similar to the effects of estrogen in females. This is because testosterone can be converted to estrogen and because PD occurs most often in older men whose endogenous androgens are in decline. Although our hypothesis was not supported, 


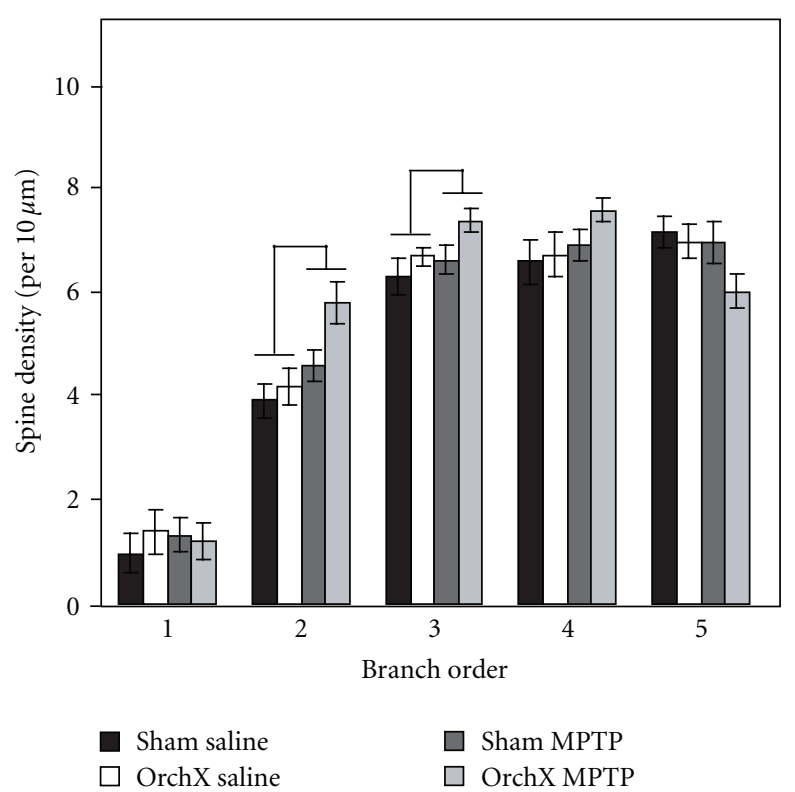

(a)

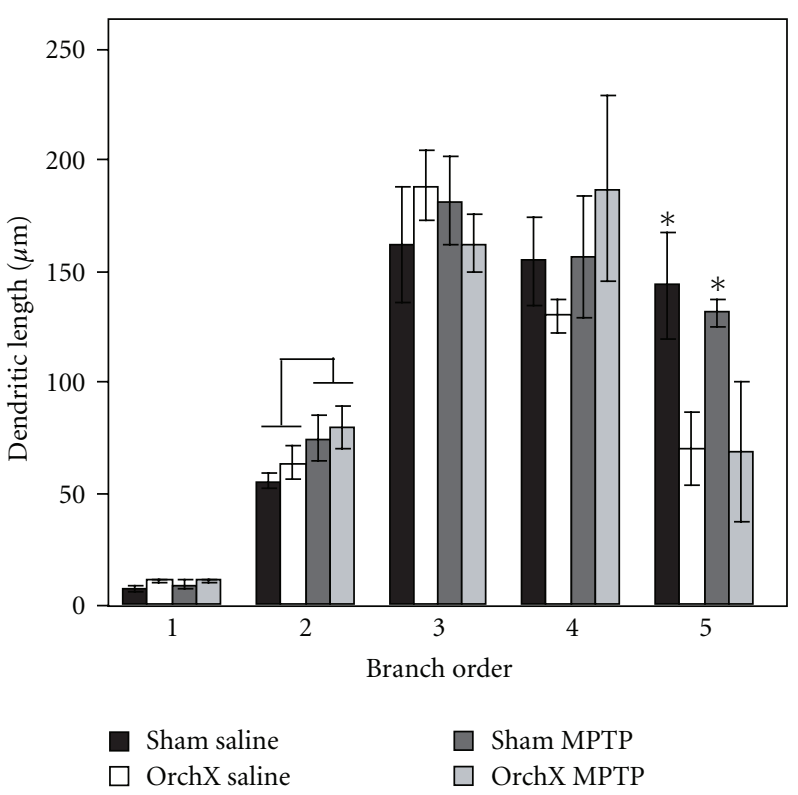

(b)

Figure 4: Branch order totals. Spine density (a) and dendritic length (b) for first- to fifth-order branches in striatal medium spiny neurons ( $n=5$ mice/group). Saline-injected mice include gonad-intact (black bars) and orchidectomized (white bars) males. MPTP-treated mice include gonad-intact (dark gray bars) and orchidectomized (light gray bars) males. Bars represent an effect of MPTP $(P<.05)$, and asterisks represent an effect of gonadectomy $(P<.05)$. Abbreviations: OrchX, orchidectomized; Sham, sham-orchidectomized.

our findings are in agreement with previous studies which found no effect of castration on striatal dopamine loss after MPTP [18] or on TH neurons in SNC after 6-OHDA [33]. In addition, studies using methamphetamine, which also depletes striatal dopamine, have shown that dopamine depletion after methamphetamine as well as amphetamineinduced stereotyped behaviors are similar in gonad-intact and castrated animals [49-52]. Interestingly, other studies have reported that testosterone increases neurotoxicity after dopamine-depleting lesions [13, 21, 53]. Due to the tremendous variability among published studies, these results are difficult to interpret. However, testosterone does not appear to have neuroprotective effects in the male nigrostriatal system.

Despite having no effect on MPTP-induced morphologic changes, castration decreased MSN dendritic length, but this effect was restricted to the distal branches of the dendritic tree. These effects of castration suggest that testosterone promotes dendritic growth in striatal MSNs. This raises an important question. How does testosterone promote growth of distal dendrites, but fail to attenuate MPTPinduced structural remodeling? To understand this issue, it is important to understand how hormones modulate neuronal plasticity, where hormones act in relation to the striatum and, in the case of testosterone, whether they act via androgenic or estrogenic mechanisms.

In hormone-sensitive areas of the brain, testosteronedriven changes in structural morphology are driven by classical hormone receptors for androgen or estrogen. For example, castration decreases dendritic branching in the posterior medial amygdala $(\mathrm{MeP})$ and medial preoptic area
(MPOA), and reduces spine density on hippocampal CA1 neurons in adult rodents $[31,54,55]$. The findings in the current study are similar. However, while MeP, MPOA and CA1 each contains an abundance of classical receptors for both androgens and estrogen, the male mouse striatum is largely devoid of classical hormone receptors [56]. Moreover, classical hormone receptors are also sparse in the major dopaminergic input to MSN dendrites from SNC [56]. Although previous studies in rats and mice have found some evidence of AR and ER in SNC [56-58], it appears that few steroid-sensitive neurons are $\mathrm{TH}$-positive and project to the striatum $[56,58]$. This relative absence of hormone receptors severely limits the ability of testosterone to exert direct or indirect effects on MSN dendrites via classical mechanisms. It is more likely that testosterone acts through nonclassical mechanisms to drive changes in the striatum, as suggested previously $[56,59]$. The present results extend this potential mechanism to include morphologic changes as well.

In the brain, testosterone can act as an androgen, but it can also act via estrogenic mechanisms after aromatization. The ability of gonadal hormones to attenuate nigrostriatal neurotoxicity is largely attributed to estrogen, which is neuroprotective in the female. In animal models, estrogen attenuates striatal dopamine depletion $[9,60,61]$ and partially prevents the loss of striatal TH immunoreactivity [14, 62] after MPTP. Indeed, experimental evidence has shown that estrogen also protects against nigrostriatal degeneration in the male striatum. However, while circulating estrogen is abundant in females, striatal estrogen available in males is minimal due to the low levels of aromatase in the striatum [63-66]. Therefore, the inability of testosterone 
to act through estrogenic mechanisms effectively prevents hormone-driven neuroprotection in the striatum. However, it does not influence the neurotrophic actions of testosterone on MSN distal dendrites, which likely occur via androgenic mechanisms.

Clinically, the actions of gonadal steroid hormones in the nigrostriatal system are important because estrogen is thought to be neuroprotective in several neurological disorders, including Parkinson's disease. In fact, women are more likely to develop PD after hysterectomy or menopause, when endogenous estrogen is eliminated [67, 68]. One of the potential benefits of hormone replacement therapy on postmenopausal women is the potential to delay the onset and/or decrease the severity of neurodegenerative disease. Men also experience a loss of testosterone with age, albeit less severe than the complete loss of gonadal steroids in the female. Although androgen replacement therapy is available for men with hypoandrogenism, the results of the current study suggest that androgen replacement will not attenuate nigrostriatal neurodegeneration in the male.

\section{Abbreviations}

6-OHDA: 6-hydroxydopamine

CA1: $\quad$ CA1 region of the hippocampus

D1: $\quad$ Dopamine receptor D1

D2: $\quad$ Dopamine receptor D2

MeP: $\quad$ Posterior medial amygdala

MPOA: Medial preoptic area of the hypothalamus

MPTP: 1-methyl-4-phenyl-1,2,3,6-tetrahydropyridine

MSN: Medium spiny neuron

PD: $\quad$ Parkinson's disease

SNC: $\quad$ Substantia nigra pars compacta

TH: $\quad$ Tyrosine hydroxylase.

\section{Acknowledgments}

The authors thank Thomas McNeill for the use of his imaging system to analyze Golgi-stained neurons. In addition, they thank Marta Vuckovic, Elizabeth Hogg, and Christina Zeytuntsyan for their assistance on this project. This work was supported by NIH K18-DC009125 to R. I. Wood, NIH RO1-NS044327 to M. W. Jakowec, and the USC Final Year Dissertation Fellowship to E. Antzoulatos.

\section{References}

[1] L. M. Garcia-Segura, J. A. Chowen, A. Parducz, and F. Naftolin, "Gonadal hormones as promoters of structural synaptic plasticity: cellular mechanisms," Progress in Neurobiology, vol. 44, no. 3, pp. 279-307, 1994.

[2] L. M. Garcia-Segura, I. Azcoitia, and L. L. DonCarlos, "Neuroprotection by estradiol," Progress in Neurobiology, vol. 63 , no. 1, pp. 29-60, 2001.

[3] M. Białek, P. Zaremba, K. K. Borowicz, and S. J. Czuczwar, "Neuroprotective role of testosterone in the nervous system," Polish Journal of Pharmacology, vol. 56, no. 5, pp. 509-518, 2004.
[4] L. M. Shulman and V. Bhat, "Gender disparities in Parkinson's disease," Expert Review of Neurotherapeutics, vol. 6, no. 3, pp. 407-416, 2006.

[5] S. Fahn, "Description of Parkinson's disease as a clinical syndrome," Annals of the New York Academy of Sciences, vol. 991, pp. 1-14, 2003.

[6] J. B. Becker, "Oestrogen effects on dopaminergic function in striatum," Novartis Foundation Symposium, vol. 230, pp. 134151, 2000.

[7] D. E. Dluzen, "Neuroprotective effects of estrogen upon the nigrostriatal dopaminergic system," Journal of Neurocytology, vol. 29, no. 5-6, pp. 387-399, 2000.

[8] A. Schober, "Classic toxin-induced animal models of Parkinson's disease: 6-OHDA and MPTP," Cell and Tissue Research, vol. 318, no. 1, pp. 215-224, 2004.

[9] D. E. Dluzen, J. L. McDermott, and B. Liu, "Estrogen as a neuroprotectant against MPTP-induced neurotoxicity in C57/B1 mice," Neurotoxicology and Teratology, vol. 18, no. 5, pp. 603-606, 1996.

[10] K. A. Disshon and D. E. Dluzen, "Estrogen as a neuromodulator of MPTP-induced neurotoxicity: effects upon striatal dopamine release," Brain Research, vol. 764, no. 1-2, pp. 9-16, 1997.

[11] D. Dluzen, "Estrogen decreases corpus striatal neurotoxicity in response to 6- hydroxydopamine," Brain Research, vol. 767, no. 2, pp. 340-344, 1997.

[12] D. B. Miller, S. F. Ali, J. P. O’Callaghan, and S. C. Laws, "The impact of gender and estrogen on striatal dopaminergic neurotoxicity," Annals of the New York Academy of Sciences, vol. 844, pp. 153-165, 1998.

[13] H. E. Murray, A. V. Pillai, S. R. Mcarthur et al., "Dose- and sex-dependent effects of the neurotoxin 6-hydroxydopamine on the nigrostriatal dopaminergic pathway of adult rats: differential actions of estrogen in males and females," Neuroscience, vol. 116, no. 1, pp. 213-222, 2003.

[14] P. J. Shughrue, "Estrogen attenuates the MPTP-induced loss of dopamine neurons from the mouse SNc despite a lack of estrogen receptors (ER $\alpha$ and $\operatorname{ER} \beta)$," Experimental Neurology, vol. 190, no. 2, pp. 468-477, 2004.

[15] A. C. Ferraz, F. Matheussi, R. E. Szawka et al., "Evaluation of estrogen neuroprotective effect on nigrostriatal dopaminergic neurons following 6-hydroxydopamine injection into the substantia nigra pars compacta or the medial forebrain bundle," Neurochemical Research, vol. 33, no. 7, pp. 1238-1246, 2008.

[16] J. B. Becker, "Gender differences in dopaminergic function in striatum and nucleus accumbens," Pharmacology Biochemistry and Behavior, vol. 64, no. 4, pp. 803-812, 1999.

[17] M. Bourque, D. E. Dluzen, and T. Di Paolo, "Neuroprotective actions of sex steroids in Parkinson's disease," Frontiers in Neuroendocrinology, vol. 30, no. 2, pp. 142-157, 2009.

[18] D. E. Dluzen, "Effects of testosterone upon MPTP-induced neurotoxicity of the nigrostriatal dopaminergic system of C57/B1 mice," Brain Research, vol. 715, no. 1-2, pp. 113-118, 1996.

[19] A. Ekue, J. F. Boulanger, M. Morissette, and T. Di Paolo, "Lack of effect of testosterone and dihydrotestosterone compared to $17 \beta$-oestradiol in 1-methyl-4-phenyl1,2,3,6,tetrahydropyridine-mice," Journal of Neuroendocrinology, vol. 14, no. 9, pp. 731-736, 2002.

[20] D. Dluzen, R. Jain, and B. Liu, "Modulatory effects of testosterone on 1-methyl-4-phenyl-1,2,3,6- tetrahydropyridineinduced neurotoxicity," Journal of Neurochemistry, vol. 62, no. 1, pp. 94-101, 1994. 
[21] G. E. Gillies, H. E. Murray, D. Dexter, and S. McArthur, "Sex dimorphisms in the neuroprotective effects of estrogen in an animal model of Parkinson's disease," Pharmacology Biochemistry and Behavior, vol. 78, no. 3, pp. 513-522, 2004.

[22] V. Jackson-Lewis, M. Jakowec, R. E. Burke, and S. Przedborski, "Time course and morphology of dopaminergic neuronal death caused by the neurotoxin 1-methyl-4-phenyl-1,2,3,6tetrahydropyridine," Neurodegeneration, vol. 4, no. 3, pp. 257269, 1995.

[23] M. W. Jakowec, K. Nixon, E. Hogg, T. McNeill, and G. M. Petzinger, "Tyrosine hydroxylase and dopamine transporter expression following 1-methyl-4-phenyl-1,2,3,6-tetrahydropyridine-induced neurodegeneration of the mouse nigrostriatal pathway," Journal of Neuroscience Research, vol. 76, no. 4, pp. 539-550, 2004.

[24] G. Paxinos and K. Franklin, The Mouse Brain in Stereotaxic Coordinates, Academic Press, New York, NY, USA, 2001.

[25] B. E. Fisher, G. M. Petzinger, K. Nixon et al., "Exercise-induced behavioral recovery and neuroplasticity in the 1-methyl-4phenyl-1,2,3,6-tetrahydropyridine-lesioned mouse basal ganglia," Journal of Neuroscience Research, vol. 77, no. 3, pp. 378390, 2004.

[26] G. M. Petzinger, J. P. Walsh, G. Akopian et al., "Effects of treadmill exercise on dopaminergic transmission in the 1-methyl-4phenyl-1,2,3,6-tetrahydropyridine-lesioned mouse model of basal ganglia injury," Journal of Neuroscience, vol. 27, no. 20, pp. 5291-5300, 2007.

[27] D. M. Gomez and S. W. Newman, "Medial nucleus of the amygdala in the adult syrian hamster: a quantitative golgi analysis of gonadal hormonal regulation of neuronal morphology," Anatomical Record, vol. 231, no. 4, pp. 498-509, 1991.

[28] R. Gibb and B. Kolb, "A method for vibratome sectioning of Golgi-Cox stained whole rat brain," Journal of Neuroscience Methods, vol. 79, no. 1, pp. 1-4, 1998.

[29] R. Kurosaki, Y. Muramatsu, H. Watanabe et al., "Role of dopamine transporter against MPTP (1-methyl-4-phenyl1,2,3,6-tetrahydropyridine) neurotoxicity in mice," Metabolic Brain Disease, vol. 18, no. 2, pp. 139-146, 2003.

[30] M. M. Monaghan, L. Leddy, M. -L. A. Sung et al., "Social odor recognition: a novel behavioral model for cognitive dysfunction in Parkinson's disease," Neurodegenerative Diseases, vol. 7, no. 1-3, pp. 153-159, 2010.

[31] E. Petroske, G. E. Meredith, S. Callen, S. Totterdell, and Y. S. Lau, "Mouse model of Parkinsonism: a comparison between subacute MPTP and chronic MPTP/probenecid treatment," Neuroscience, vol. 106, no. 3, pp. 589-601, 2001.

[32] M. G. Vučković, R. I. Wood, D. P. Holschneider et al., "Memory, mood, dopamine, and serotonin in the 1-methyl-4phenyl-1,2,3,6-tetrahydropyridine-lesioned mouse model of basal ganglia injury," Neurobiology of Disease, vol. 32, no. 2, pp. 319-327, 2008.

[33] S. McArthur, H. E. Murray, A. Dhankot, D. T. Dexter, and G. E. Gillies, "Striatal susceptibility to a dopaminergic neurotoxin is independent of sex hormone effects on cell survival and DAT expression but is exacerbated by central aromatase inhibition," Journal of Neurochemistry, vol. 100, no. 3, pp. 678-692, 2007.

[34] M. Day, Z. Wang, J. Ding et al., "Selective elimination of glutamatergic synapses on striatopallidal neurons in Parkinson disease models," Nature Neuroscience, vol. 9, no. 2, pp. 251259,2006
[35] S. Pulipparacharuvil, W. Renthal, C. F. Hale et al., "Cocaine regulates MEF2 to control synaptic and behavioral plasticity," Neuron, vol. 59, no. 4, pp. 621-633, 2008.

[36] C. A. Vickers, B. Stephens, J. Bowen, G. W. Arbuthnott, S. G. N. Grant, and C. A. Ingham, "Neurone specific regulation of dendritic spines in vivo by post synaptic density 95 protein (PSD-95)," Brain Research, vol. 1090, no. 1, pp. 89-98, 2006.

[37] K. E. Lyons, J. P. Hubble, A. I. Tröster, R. Pahwa, and W. C. Koller, "Gender differences in Parkinson's disease," Clinical Neuropharmacology, vol. 21, no. 2, pp. 118-121, 1998.

[38] C. A. Haaxma, B. R. Bloem, G. F. Borm et al., "Gender differences in Parkinson's disease," Journal of Neurology, Neurosurgery and Psychiatry, vol. 78, no. 8, pp. 819-824, 2007.

[39] E. Antzoulatos, M. W. Jakowec, G. M. Petzinger, and R. I. Wood, "Sex differences in motor behavior in the MPTP mouse model of Parkinson's disease," Pharmacology Biochemistry and Behavior, vol. 95, no. 4, pp. 466-472, 2010.

[40] M. D. Neely, D. E. Schmidt, and A. Y. Deutch, "Cortical regulation of dopamine depletion-induced dendritic spine loss in striatal medium spiny neurons," Neuroscience, vol. 149, no. 2, pp. 457-464, 2007.

[41] A. D. Smith and J. P. Bolam, "The neural network of the basal ganglia as revealed by the study of synaptic connections of identified neurones," Trends in Neurosciences, vol. 13, no. 7, pp. 259-265, 1990.

[42] I. N. Krasnova and J. L. Cadet, "Methamphetamine toxicity and messengers of death," Brain Research Reviews, vol. 60, no. 2, pp. 379-407, 2009.

[43] Y. Li, B. Kolb, and T. E. Robinson, "The location of persistent amphetamine-induced changes in the density of dendritic spines on medium spiny neurons in the nucleus accumbens and caudate-putamen," Neuropsychopharmacology, vol. 28, no. 6, pp. 1082-1085, 2003.

[44] J. P. Jedynak, J. M. Uslaner, J. A. Esteban, and T. E. Robinson, "Methamphetamine-induced structural plasticity in the dorsal striatum," European Journal of Neuroscience, vol. 25, no. 3, pp. 847-853, 2007.

[45] T. E. Robinson and B. Kolb, "Structural plasticity associated with exposure to drugs of abuse," Neuropharmacology, vol. 47, no. 1, pp. 33-46, 2004.

[46] J. H. Baik, R. Picetti, A. Saiardi et al., "Parkinsonianlike locomotor impairment in mice lacking dopamine D2 receptors," Nature, vol. 377, no. 6548, pp. 424-428, 1995.

[47] M. Xu, Y. Guo, C. V. Vorhees, and J. Zhang, "Behavioral responses to cocaine and amphetamine administration in mice lacking the dopamine D1 receptor," Brain Research, vol. 852, no. 1, pp. 198-207, 2000.

[48] J. Drago, C. R. Gerfen, J. E. Lachowicz et al., "Altered striatal function in a mutant mouse lacking $\mathrm{D}(1 \mathrm{~A})$ dopamine receptors," Proceedings of the National Academy of Sciences of the United States of America, vol. 91, no. 26, pp. 12564-12568, 1994.

[49] D. M. Camp, J. B. Becker, and T. E. Robinson, "Sex differences in the effects of gonadectomy on amphetamine-induced rotational behavior in rats," Behavioral and Neural Biology, vol. 46, no. 3, pp. 491-495, 1986.

[50] X. Gao and D. E. Dluzen, "The effect of testosterone upon methamphetamine neurotoxicity of the nigrostriatal dopaminergic system," Brain Research, vol. 892, no. 1, pp. 6369, 2001.

[51] D. E. Dluzen, L. I. Anderson, and C. F. Pilati, "Methamphetamine-gonadal steroid hormonal interactions: effects 
upon acute toxicity and striatal dopamine concentrations," Neurotoxicology and Teratology, vol. 24, no. 2, pp. 267-273, 2002.

[52] R. E. Myers, L. I. Anderson, and D. E. Dluzen, "Estrogen, but not testosterone, attenuates methamphetamine-evoked dopamine output from superfused striatal tissue of female and male mice," Neuropharmacology, vol. 44, no. 5, pp. 624-632, 2003.

[53] C. Lewis and D. E. Dluzen, “Testosterone enhances dopamine depletion by methamphetamine in male, but not female, mice," Neuroscience Letters, vol. 448, no. 1, pp. 130-133, 2008.

[54] C. Leranth, O. Petnehazy, and N. J. MacLusky, "Gonadal hormones affect spine synaptic density in the CA1 hippocampal subfield of male rats," Journal of Neuroscience, vol. 23, no. 5, pp. 1588-1592, 2003.

[55] J. A. Cherry, S. A. Tobet, T. J. DeVoogd, and M. J. Baum, "Effects of sex and androgen treatment on dendritic dimensions of neurons in the sexually dimorphic preoptic/anterior hypothalamic area of male and female ferrets," Journal of Comparative Neurology, vol. 323, no. 4, pp. 577-585, 1992.

[56] P. J. Shughrue, "Estrogen attenuates the MPTP-induced loss of dopamine neurons from the mouse SNc despite a lack of estrogen receptors (ER $\alpha$ and ER $\beta)$," Experimental Neurology, vol. 190, no. 2, pp. 468-477, 2004.

[57] S. W. Mitra, E. Hoskin, J. Yudkovitz et al., "Immunolocalization of estrogen receptor $\beta$ in the mouse brain: comparison with estrogen receptor $\alpha$, Endocrinology, vol. 144, no. 5, pp. 2055-2067, 2003.

[58] M. F. Kritzer, "Selective colocalization of immunoreactivity for intracellular gonadal hormone receptors and tyrosine hydroxylase in the ventral tegmental area, substantia nigra, and retrorubral fields in the rat," Journal of Comparative Neurology, vol. 379, no. 2, pp. 247-260, 1997.

[59] E. J. Roy, D. R. Buyer, and V. A. Licari, "Estradiol in the striatum: effects on behavior and dopamine receptors but no evidence for membrane steroid receptors," Brain Research Bulletin, vol. 25, no. 2, pp. 221-227, 1990.

[60] A. D. Ramirez, X. Liu, and F. S. Menniti, "Repeated estradiol treatment prevents MPTP-induced dopamine depletion in male mice," Neuroendocrinology, vol. 77, no. 4, pp. 223-231, 2003.

[61] D. E. Dluzen, J. L. McDermott, and B. Liu, "Estrogen alters MPTP-induced neurotoxicity in female mice: effects on striatal dopamine concentrations and release," Journal of Neurochemistry, vol. 66, no. 2, pp. 658-666, 1996.

[62] M. Ookubo, H. Yokoyama, S. Takagi, H. Kato, and T. Araki, "Effects of estrogens on striatal damage after 1-methyl-4phenyl-1,2,3,6-tetrahydropyridine (MPTP) neurotoxicity in male and female mice," Molecular and Cellular Endocrinology, vol. 296, no. 1-2, pp. 87-93, 2008.

[63] E. Küppers and C. Beyer, "Expression of aromatase in the embryonic and postnatal mouse striatum," Molecular Brain Research, vol. 63, no. 1, pp. 184-188, 1998.

[64] C. E. Roselli and J. A. Resko, "Sex differences in androgenregulated expression of cytochrome $\mathrm{P} 450$ aromatase in the rat brain," Journal of Steroid Biochemistry and Molecular Biology, vol. 61, no. 3-6, pp. 365-374, 1997.

[65] C. K. Wagner and J. I. Morrell, "Distribution and steroid hormone regulation of aromatase mRNA expression in the forebrain of adult male and female rats: a cellular-level analysis using in situ hybridization," Journal of Comparative Neurology, vol. 370, no. 1, pp. 71-84, 1996.
[66] C. K. Wagner and J. I. Morrell, "Neuroanatomical distribution of aromatase mRNA in the rat brain: indications of regional regulation," Journal of Steroid Biochemistry and Molecular Biology, vol. 61, no. 3-6, pp. 307-314, 1997.

[67] P. Ragonese, M. D’Amelio, G. Salemi et al., "Risk of Parkinson disease in women: effect of reproductive characteristics," Neurology, vol. 62, no. 11, pp. 2010-2014, 2004.

[68] R. A. Popat, S. K. Van Den Eeden, C. M. Tanner et al., "Effect of reproductive factors and postmenopausal hormone use on the risk of Parkinson disease," Neurology, vol. 65, no. 3, pp. 383-390, 2005. 


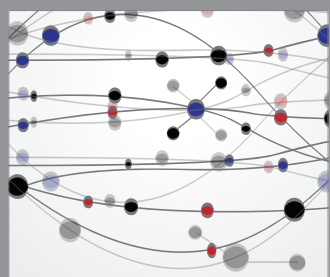

The Scientific World Journal
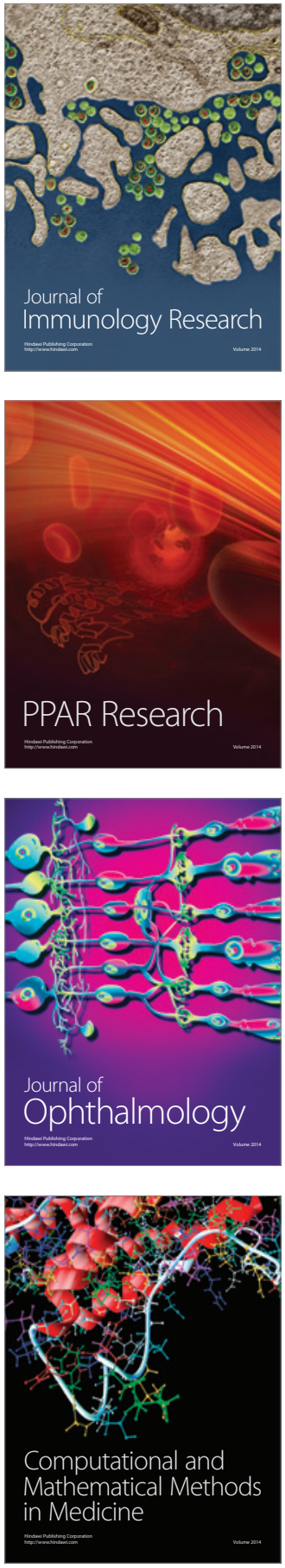

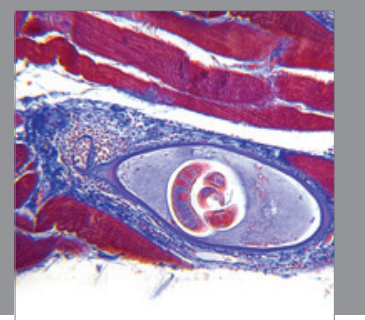

Gastroenterology

Research and Practice
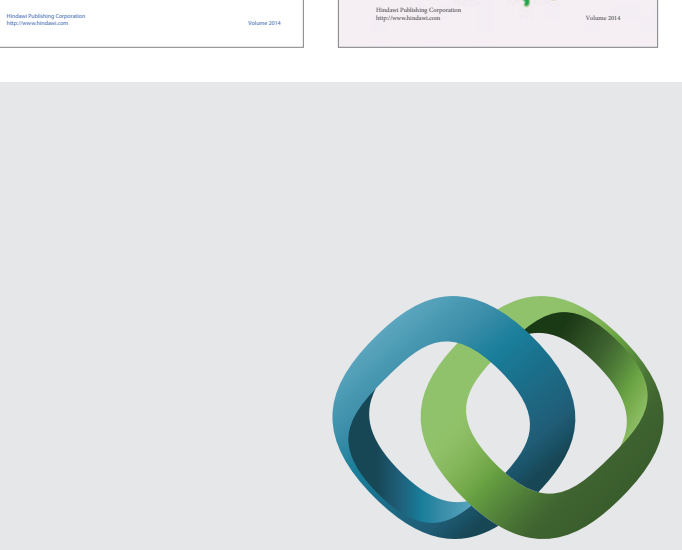

\section{Hindawi}

Submit your manuscripts at

http://www.hindawi.com
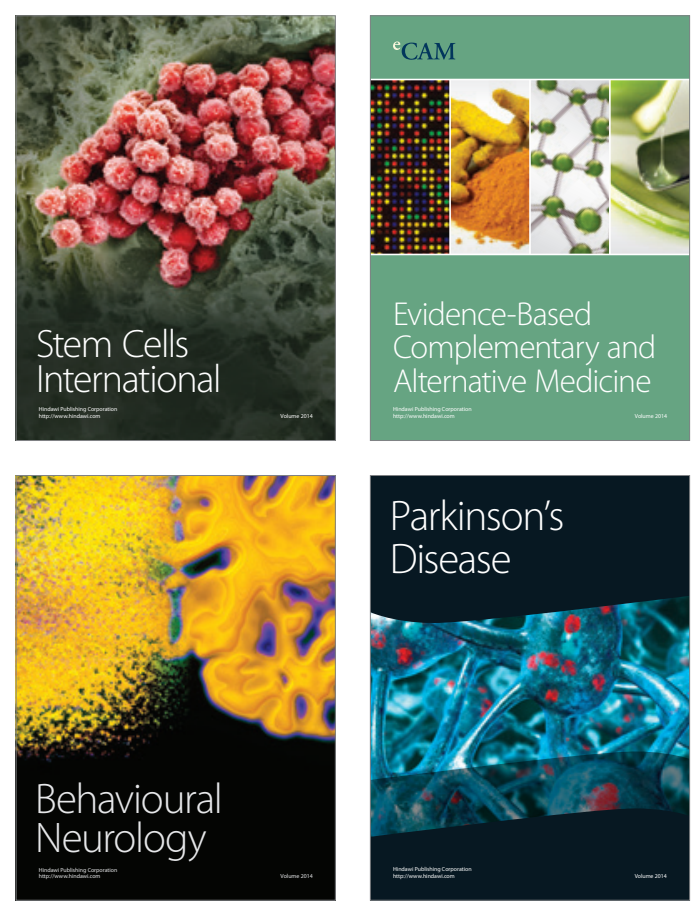

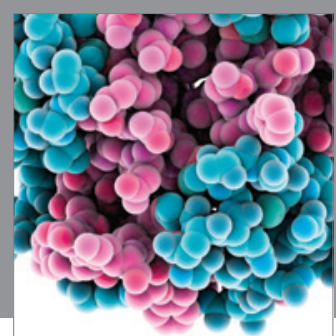

Journal of
Diabetes Research

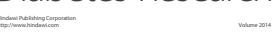

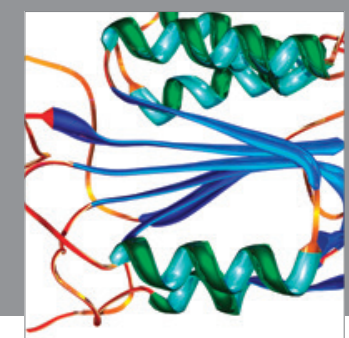

Disease Markers
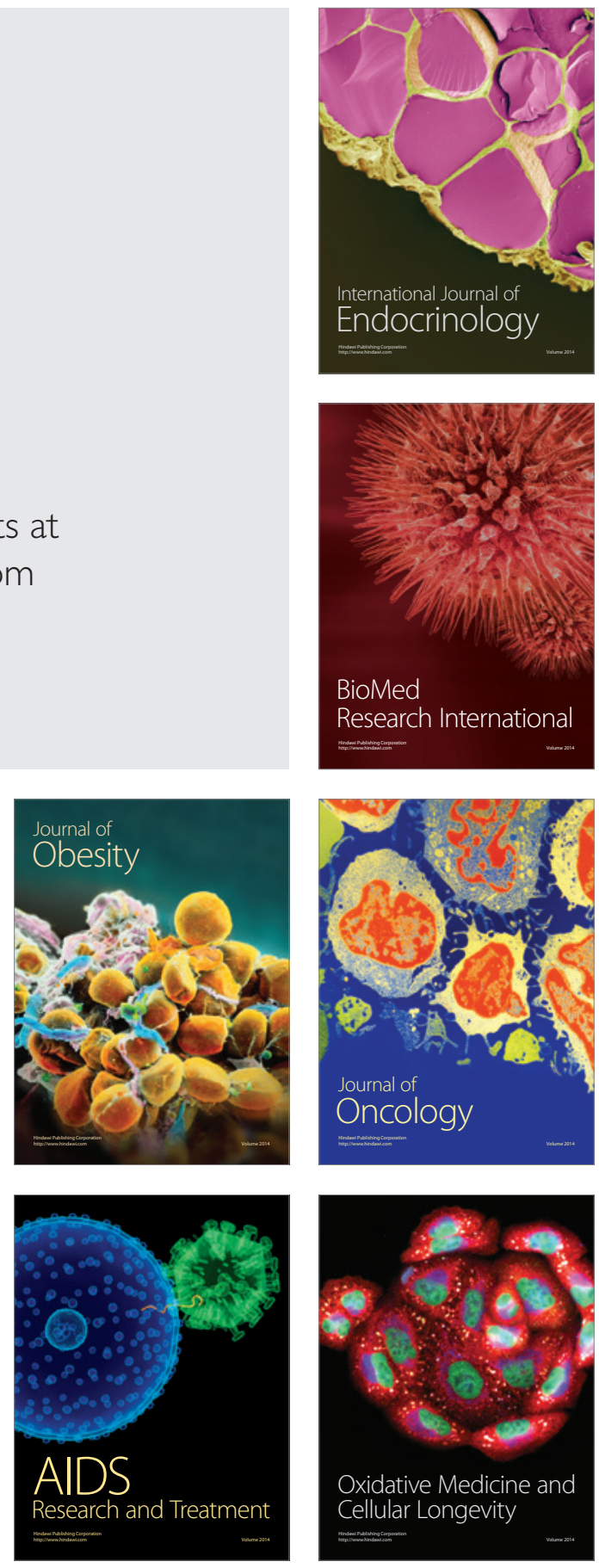